\title{
Cooperativity, Smooth Energy Landscapes and the Origins of Topology-dependent Protein Folding Rates
}

\author{
Andrew I. Jewett ${ }^{1}$, Vijay S. Pande ${ }^{2}$ and Kevin W. Plaxco ${ }^{3 *}$
}

${ }^{1}$ Department of Physics

University of California at

Santa Barbara, Santa Barbara

CA 93106, USA

${ }^{2}$ Department of Chemistry Stanford University, Stanford CA 94305, USA

${ }^{3}$ Department of Chemistry and Biochemistry, University of California at Santa Barbara Santa Barbara, CA 93106-9510 USA

\begin{abstract}
The relative folding rates of simple, single-domain proteins, proteins whose folding energy landscapes are smooth, are highly dispersed and strongly correlated with native-state topology. In contrast, the relative folding rates of small, Gō-potential lattice polymers, which also exhibit smooth energy landscapes, are poorly dispersed and insignificantly correlated with native-state topology. Here, we investigate this discrepancy in light of a recent, quantitative theory of two-state folding kinetics, the topomer search model. This model stipulates that the topology-dependence of two-state folding rates is a direct consequence of the extraordinarily cooperative equilibrium folding of simple proteins. We demonstrate that traditional Gō polymers lack the extreme cooperativity that characterizes the folding of naturally occurring, two-state proteins and confirm that the folding rates of a diverse set of Gō 27-mers are poorly dispersed and effectively uncorrelated with native state topology. Upon modestly increasing the cooperativity of the Gö-potential, however, significantly increased dispersion and strongly topology-dependent kinetics are observed. These results support previous arguments that the cooperative folding of simple, single-domain proteins gives rise to their topologydependent folding rates. We speculate that this cooperativity, and thus, indirectly, the topology-rate relationship, may have arisen in order to generate the smooth energetic landscapes upon which rapid folding can occur.
\end{abstract}

(C) 2003 Elsevier Science Ltd. All rights reserved

Keywords: contact order; non-additivity; topological frustration of short-range versus long-range contacts in the native state", play a significant role in defining the rates with which lattice polymers fold. . $^{2,4}$,

Experimental investigations, in contrast, indicate that the parameters that often account for the widely varying folding rates of simplified, proteinlike models do not necessarily account for the wide range of rates observed for the folding of real proteins. For example, while roughness on the energy landscape produces lattice polymer folding rates varying by orders of magnitude, ${ }^{6}$ the folding energy landscape of most simple proteins is exceedingly smooth. ${ }^{7}$ Similarly, despite the strong correlation between the equilibrium collapse parameter $\sigma$ and the folding rates of simple on-lattice and off-lattice protein models, ${ }^{3}$ no correlation is observed between $\sigma$ and folding rates in the laboratory. ${ }^{8}$ Instead, topology, as defined by several measures of the number of short-range versus long-range contacts in the native state, largely defines the relative folding rates of simple, single-domain proteins..$^{9-13}$

The topology-dependent folding kinetics of simple proteins might be coupled to the
Abbreviations used: MFPT, mean first passage time.

E-mail address of the corresponding author: kwp@chem.ucsb.edu 


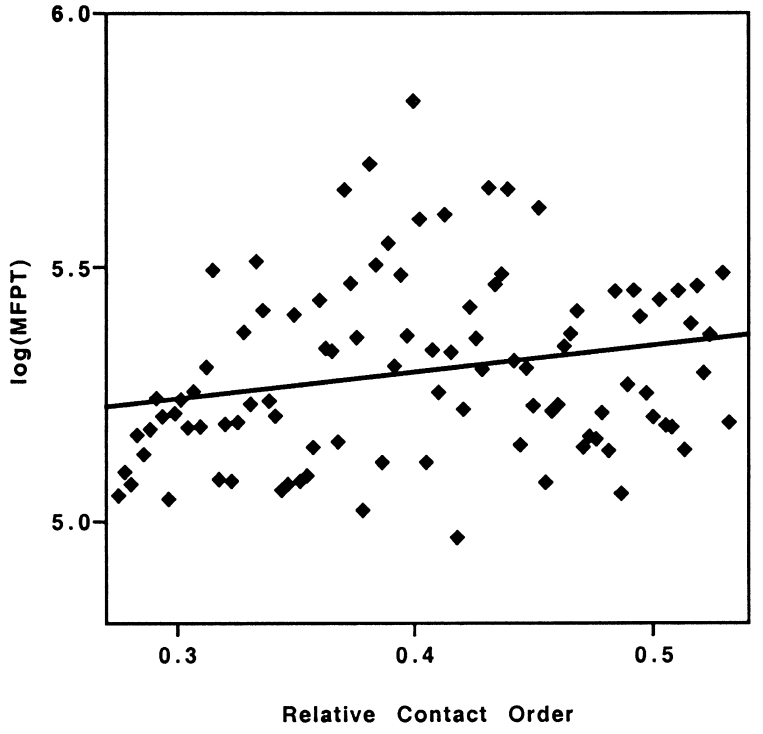

Figure 1. The folding kinetics (as mean first passage time $\approx 1 / k_{\mathrm{f}}$ ) of traditional Gō 27 -mers are poorly dispersed and insignificantly correlated $\left(r^{2}=0.05\right.$; $p=0.06$ ) with measures of native state topology such as contact order. The smooth energy landscapes associated with Gō lattice polymers are apparently insufficient to generate topology-dependence in their folding rates.

smoothness of their energy landscapes; if the relevant topological effects are subtle, they might be apparent only when the potentially larger kinetic effects arising from energetic roughness (i.e. partially folded or misfolded traps) are eliminated. This argument suggests that, in the absence of rate dispersion induced by energetic roughness, the folding rates of lattice polymers might similarly correlate with native-state topology. The energy landscapes of Gō-potential polymers, polymers in which only interactions present in the native state are favorable, ${ }^{14}$ are relatively smooth and thus provide a means of testing this hypothesis. Extensive simulations of the folding of Gō polymers, however, demonstrate that smooth energy landscapes alone do not generate strongly topologydependent folding rates; on-lattice Gō polymer folding rates are effectively uncorrelated with topology (M. Cieplak, personal communication) and the topology-dependence of more sophisticated, off-lattice Gō polymers ranges from moderate ${ }^{15}$ to non-existent. ${ }^{16}$ No simple polymer model described to date, no matter how smooth the energy landscape, exhibits the dramatic, many orders of magnitude topology-dependence observed during the folding of simple, singledomain proteins.

Because the folding energy landscapes of simple, two-state proteins and small, Gō polymer models are both smooth, other parameters must account for the significant discrepancy in the topologydependence of their folding rates. A possible candidate for the origins of this discrepancy is cooperativity: the phenomenon that the breaking of (non-covalent) native-state interactions lowers the energetic barrier to the breaking of additional interactions, producing a non-linear relationship between free energy and the number of native contacts. In comparison to proteins, the folding of traditional Gō polymers is relatively noncooperative. ${ }^{17}$ For example, as shown here, the free energy of partially structured Gō lattice polymers is a relatively linear function of the total number of interactions present, whereas the free energy of partially folded proteins falls precipitously only as the last few residues adopt the native

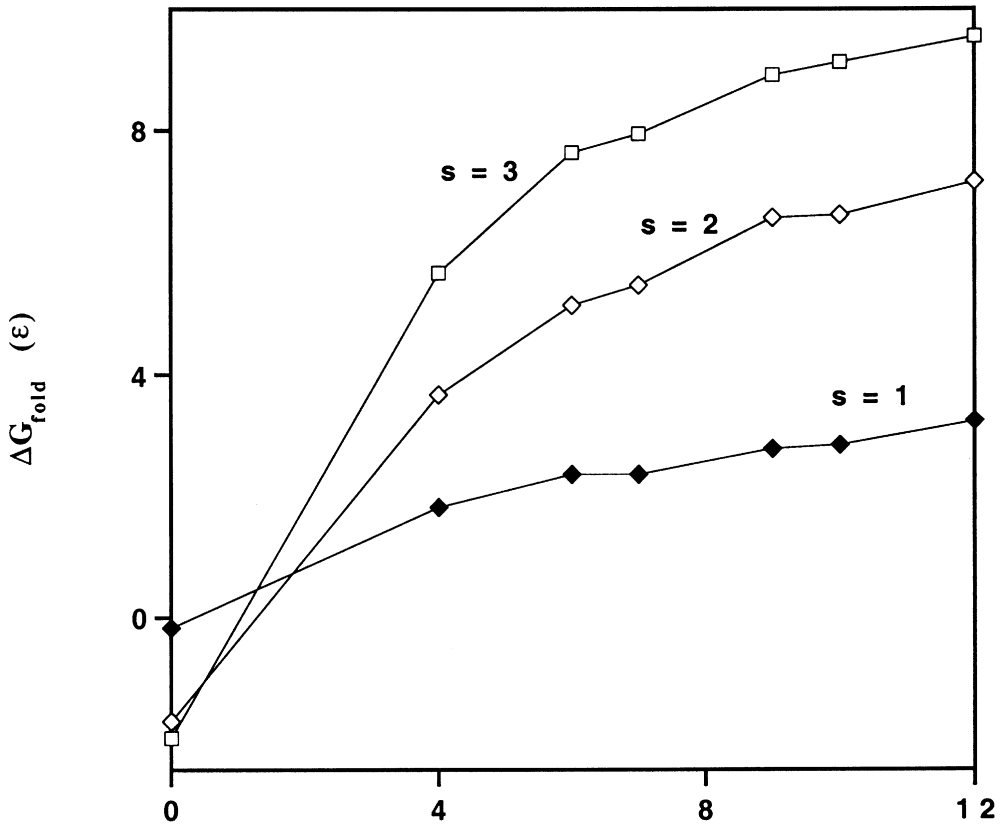

Number of Contacts Deleted
Figure 2. The folding free energy of simple, single-domain proteins rises catastrophically when as few as one, two or three residues are removed from either terminus. ${ }^{17-19}$ The folding free energy of traditional Gō polymers (filled symbols), in contrast, is a weaker, relatively linear function of the number of native interactions. Shown here is the folding free energy of a typical Gō polymer (structure with median contact order; $s=1$ ) as terminal residues are removed sequentially. If the energy of each structure is scaled as a non-linear, "cooperative" function of the number of native contacts formed (see Figure 3), significantly more protein-like folding behavior is observed (open symbols). Shown here is the change in folding free energy upon truncation for the same polymer structure with the cooperativity factor $s$ set to 2 or 3 . 


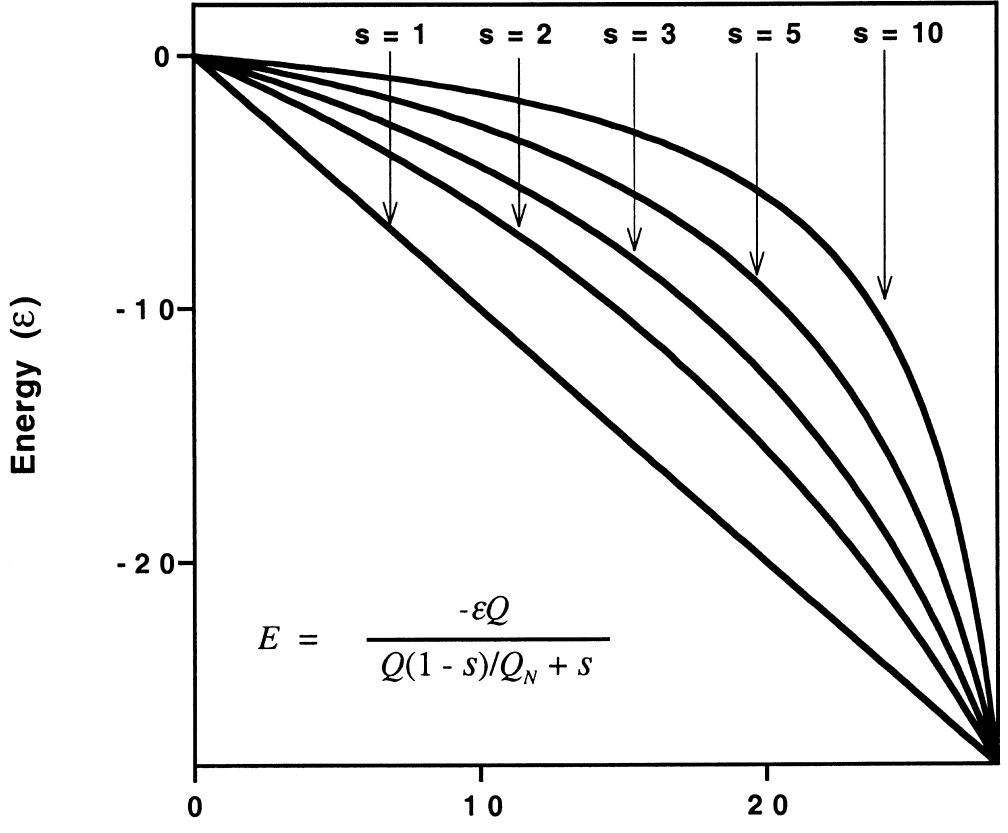

Number of Native Contacts
Figure 3. An illustration of the non-linear cooperativity function employed in this study. In order to generate arbitrary levels of cooperativity, the traditional Gō energy $(E=-\varepsilon Q$; where $Q$ is the number of native contacts formed and $-\varepsilon$ is the contact energy) is scaled by a non-linear function (equation (1); inserted). The diagonal, $s=1$, represents a traditional, non-cooperative Gō polymer in which the energy is proportional to the number of native interactions. Here, we explore the folding kinetics of this traditional Gō potential and the more cooperative, more proteinlike $s=2$ and $s=3$ modified Gō potentials. conformation (the truncation of one to three residues from the structured termini of small, singledomain proteins almost invariably leads to their complete unfolding). ${ }^{18-20}$ Here, we explore the relationship between cooperativity and topologydependent folding kinetics via simulations of a topologically diverse set of traditional and more cooperative Gō lattice heteropolymers.

\section{Results}

The folding rates of Gō 27-mers are poorly dispersed; the most rapidly folding structure in our data set of 97 diverse topologies folds only 7.2 times more rapidly than the slowest (Figure 1). Consistent with this limited dispersion, the folding rates of Go polymers are at best only very poorly related to measures of native-state topology: the correlation between folding rate (as defined by log of the mean first passage time-MFPT $\approx 1 / k_{\mathrm{f}}$ ) and contact order, for example, is statistically insignificant (slope $=0.52 ; r^{2}=0.05, p=0.06$ ). Clearly topology, at least as defined by contact order, is not a major determinant of the relative folding rates of Gō 27-mers.

A smooth energy landscape thus appears insufficient to generate strongly topology-dependent folding rates in simple lattice heteropolymers. What then might account for the highly topologydependent folding rates of simple proteins? Recent theory suggests that topology-dependent rates arise due to the extraordinarily cooperative equilibrium folding of small proteins. ${ }^{13}$ Critically, simulations of the folding of traditional Go polymers demonstrate that they generally lack cooperativity. ${ }^{17}$ For example, the free energy of tra- ditional Gō lattice polymers is only relatively weakly and linearly dependent on the number of native interactions formed (Figure 2).

We have tested the hypothesized linkage between cooperativity and topology-dependent kinetics via simulations of the folding of Gō lattice polymers that have been modified in order to increase their cooperativity. The requisite modification entails altering the potential function such that the energy of the polymer is, in contrast to traditional Go polymers, a non-linear function of the number of native interactions formed. In order to generate arbitrarily cooperative folding thermodynamics, we modify the Gō energy of a given conformation $(E=-\varepsilon Q)$ by a non-linear scaling factor such that:

$$
E=\frac{-\varepsilon Q}{Q(1-s) / Q_{\mathrm{N}}+s}
$$

where $\varepsilon$ is the energy of forming a native contact, $Q$ is the number of native contacts present, $Q_{N}$ is the number of contacts in the native state $(28$ for compact $3 \times 3 \times 3$ structures) and $s$ is an adjustable parameter that varies the magnitude of the induced cooperativity (Figure 3). While the extent to which this function mimics the physics underlying the cooperativity of protein folding is debatable, this approach is sufficient to address the question of whether any cooperativity is sufficient to produce topology-dependent heteropolymer folding rates. When $s=1$, the traditional, non-cooperative Gō polymer is recovered. When $s=2$, Gō 27-mers achieve both greater native-state stability and more protein-like folding cooperativity (Figure 2, open diamonds). When $s=3$, both cooperativity and native-state stability increase 

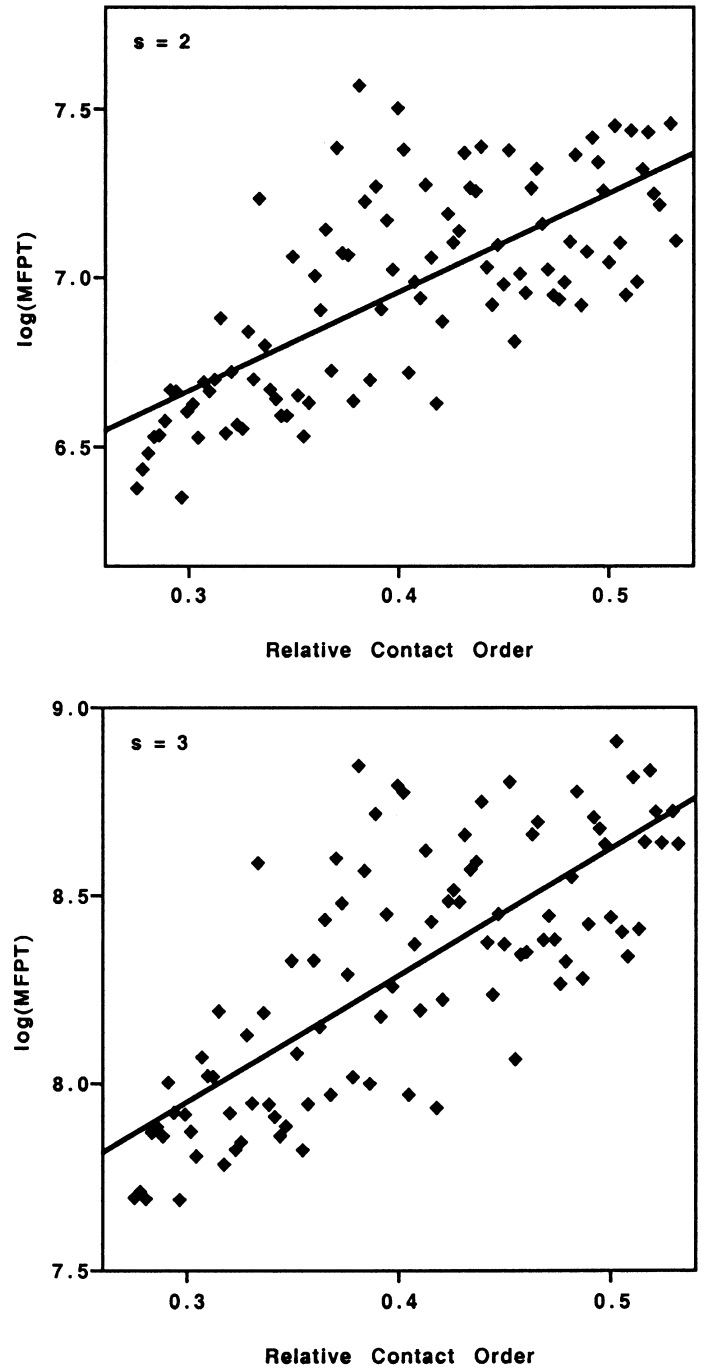

Figure 4. The addition of a small amount of cooperativity to the Gō polymer greatly increases folding rate dispersion and produces a strong, statistically significant relationship between rates and contact order. Upper: when $s=2$, the slope of the $\log (\mathrm{MFPT})$ versus contact order relationship increases 5.7 -fold relative to the traditional, non-cooperative Gō polymer $\left(r^{2}=0.51\right.$, $\left.p<10^{-16}\right)$. Lower: when $s=3$, the increase in slope rises to 6.5 -fold $\left(r^{2}=0.57 ; p<10^{-18}\right)$. In the limit of a smooth energy landscape the addition of even marginal cooperativity is sufficient to produce a topology-rate relationship analogous to, if somewhat weaker than $\left(r^{2} \approx 0.8\right)$, that observed experimentally. ${ }^{13}$

still further (Figure 2, squares), although even this level of cooperativity may be less dramatic than that observed in protein folding. ${ }^{18-20}$

We find that topology, as defined by contact order, is a major determinant of the relative folding rates of cooperative Gō lattice polymers. When $s=2$, the dispersion in folding rates increases to more than twice that of the traditional, $s=1$ case and the slope of the $\log (\mathrm{MFPT})$ versus contact order curve increases 5.7-fold (Figure 4, upper). Critically, the increase in dispersion reflects a highly significant correlation between $\log (\mathrm{MFPT})$ and contact order $\left(r^{2}=0.51 ; p<10^{-16}\right)$. The significance of the correlation increases still further when $s$ is raised to $3\left(r^{2}=0.57, p<10^{-18}\right)$, and the dispersion and slope increase 2.3-fold and 6.5-fold, respectively, relative to the, non-cooperative, $s=1$ polymer (Figure 4, lower). It thus appears that the introduction of even modest cooperativity is sufficient to generate topology-dependent lattice polymer folding rates in the limit of a smooth energy landscape.

Although it is qualitatively similar, the topology-rate relationship observed even for the $s=3$ polymer is quantitatively less dramatic and less highly correlated than that observed for the folding of two-state proteins. For example, while slope of the $-\log \left(k_{\mathrm{f}}\right)$ versus contact order relationship of two-state proteins ${ }^{10}$ is at least two orders of magnitude greater than the (effectively indistinguishable from zero) slope of the $s=1$ polymers, it is also ten times greater than that of the $s=3$ polymers. Additionally, topology captures approximately $80 \%\left(r^{2} \sim 0.8\right)$ of the variance in two-state protein folding rates ${ }^{10}$ but captures only $r^{2}=57 \%$ of the variance in, for example, the $s=3$ Go polymer set. Some of the scatter and the reduced slope observed for Gō polymers no doubt arise from issues such as topological frustration, ${ }^{21}$ the formation and stability of specific nuclei or, perhaps, the inability of lattice polymer Monte Carlo simulations to capture the complex, collective motions of proteins. Indeed, with regard to the latter issue, Koga and Takada have shown that some ${ }^{15}$ (although perhaps not most ${ }^{16}$ ) off-lattice Gō models exhibit topology-dependent folding rates even in the absence of concerted efforts to increase cooperativity, albeit with a slightly weaker correlation than even the $s=2$ polymers described here. Given the apparently monotonic relationship between the slope of the topology-rate relationship and the cooperativity factor $s$, however, it seems likely that at least some of the discrepancy in both the slope and scatter reflects the still limited cooperativity of even the $s=3$ polymer set.

\section{Discussion}

While a smooth landscape biased towards the native state may be a necessary and sufficient condition to ensure rapid heteropolymer folding, ${ }^{6}$ it is not sufficient to produce the strongly topologydependent rates specifically associated with protein folding. A simple, energetically smooth, noncooperative Gō polymer exhibits exceedingly little rate dispersion and an insignificant correlation between folding rates and measures of native-state topology. In contrast, when Gō polymers are modified such that their folding cooperativity increases even slightly, their folding rate dispersion increases considerably and the correlation between folding rates and native-state topology becomes highly statistically significant. 
The observation that topology-dependent latticepolymer folding rates are coupled to folding cooperativity is consistent with the topomer search model of protein folding. ${ }^{13}$ This theory postulates that relative barrier heights are dominated by the diffusive search for the set of unfolded conformations that share a common, global topology with the native state (i.e. are in the "native topomer"22) and that, once this is achieved, the rate-limiting step has been surmounted and specific native contacts rapidly zipper to form the native state. Cooperativity is thus the reason why the topomer search process dominates folding rates; were folding less cooperative, rates would be dominated by escape from stable, native-like or misfolded states rather than by the difficulty of finding those rare, unfolded conformations that are sufficiently native-like that they can zipper the large amount of structure required before the free energy drops significantly. Similarly, while lattice polymers cannot fold via a strictly topomer-search process (on a coarse lattice it is effectively impossible to be in the native topology without actually being in the native state), topological issues, rather than the stability of misfolded traps or nucleation events, increasingly dominate Gō polymer folding rates as cooperativity is enhanced. Our simulations thus highlight the more general observation that, as cooperativity is increased, global properties such as native-state topology will play an increasing role in defining the folding barrier. ${ }^{17,23}$

These considerations lead naturally to the question of the origins of the discrepancy between the extraordinary cooperativity of protein folding and the much lesser cooperativity of lattice polymer folding. While several theories have been put forward regarding the origins of the cooperative folding of proteins, ${ }^{24}$ recent years have seen some consensus build for the suggestion that it arises due to three-body interactions related to the nature of the hydrophobic effect. ${ }^{25-27}$ Fernandez, for example, has recently demonstrated that the inclusion of implicit solvent and three-body correlations is sufficient to produce protein-like cooperativity in the equilibrium folding of a Gō lattice polymer. $^{28}$ The vast majority of reported lattice polymer potentials, in contrast, do not include such terms, which may account for their relatively non-cooperative folding.

In addition to producing realistic topologydependent kinetics, however, the addition of cooperativity also decelerates the folding of Gō polymers $^{17}$ (compare Figure 1 with Figure 4 ). Why then, might cooperatively be related to the rapid folding of proteins (where it presumably would also slow folding relative to a hypothetical, Gōlike energy landscape)? An important consideration is that Go polymers are non-physical. That is, the same forces that favor native interactions often favor non-native interactions and thus, in contrast to polymers in silico, the native-state interactions of real heteropolymers cannot be strengthened arbitrarily without increasing the roughness of the energy landscape. Cooperativity, in contrast, provides a means of destabilizing partially structured, misfolded states relative to the native fold ${ }^{17}$ and thus potentially provides a means of simultaneously smoothing the energy landscape while stabilizing the native state. This potential net win, that cooperativity accelerates folding rates more by smoothing the landscape than it decelerates them by destabilizing potentially productive intermediates, may provide a selective advantage for cooperatively folding proteins. This would suggest that the observed topological dependence of protein folding rates is a consequence of the cooperativity necessary to ensure a smooth, biased energy landscape upon which rapid folding can occur.

\section{Methods}

There are 103,346 compact, self-avoiding $3 \times 3 \times 3$ structures that a lattice polymer can adopt, each of which assumes one of 97 possible relative contact orders. (Relative contact order is defined as the mean separation between all pairs of contacting monomers divided by the length of the polymer. It is highly correlated with other, potentially relevant measures of topology, including the number of sequence-distant native pairs, and thus serves as a convenient proxy for them.) We generated a representative, topologically diverse set of 97 structures by randomly selecting one fold as representative of each contact order. All simulations were conducted at a temperature of $T=0.68\left(\varepsilon / k_{\mathrm{B}}\right)$. Control simulations indicate that this temperature is at or just below the folding transition temperatures $\left(T_{\mathrm{f}}\right.$ or $\left.T_{\mathrm{m}}\right)$ and significantly above the glass transition temperatures $\left(T_{\mathrm{g}}\right)$ of all members of the test set. Reported (Patterson) correlation coefficients are from least-squares linear fits. Reported $p$-values reflect the probability that, given the size of the data set, a slope as great or greater in magnitude than that reported would be observed by chance alone.

\section{Folding rate determinations}

Folding rates were determined as the MFPT of 1000 , 200 or 100 Monte Carlo simulations for $s=1,2$ or 3 respectively. All simulations were run until the polymer reached the folded state (i.e. without truncation at long simulation times). For all polymers, the observed ratio of the geometric mean deviation of the folding times to the MFPT is within a few percentage units of unity (data not shown), indicating that the folding process is well described as single-exponential. MPFT is thus equivalent to the folding time constant, $\tau=1 / k_{\mathrm{f}}$.

\section{Move set dependence}

Folding rates derived from Monte Carlo simulations are dependent on the move-set employed. ${ }^{29} \mathrm{~A}$ commonly employed set is limited to corner-flip, end-move and crankshaft moves that translate one or two monomers per iteration. ${ }^{30}$ This move set, however, is not ergodic; it cannot reach all 103,346 compact, cubic structures from any arbitrary "unfolded" conformation. ${ }^{31}$ In order to minimize this potential problem, and to better approximate the collective motions possible during the folding of proteins, our move set translates five consecutive monomers per iteration. This was performed by 
randomly selecting moves from the set of all possible, self-avoiding, five-monomer structures. We note, however, that control studies using the more traditional Verdier \& Stockmayer one and two residue move set at $s=1$ and $s=2$ produce results effectively identical with those presented here (data not shown).

\section{Equilibrium cooperativity}

In order to illustrate the cooperativity of the modified Go polymer, we studied the equilibrium properties of a representative structure (the structure with the median contact order). The folding free energies of the native structure and equivalent structures with one or more terminal residues removed were determined by measuring the MFPT in both the folding and unfolding directions for at least 30 transitions for each construct. This number of simulations provides a measure of free energy as $k_{\mathrm{B}} T \ln \left(\mathrm{MFPT}_{\mathrm{f}} / \mathrm{MFPT}_{\mathrm{u}}\right)$, accurate to typically well within $10 \%$ (data not shown). $Q_{\mathrm{N}}$ was held fixed at 28 contacts. Illustrated in the Figure is a series of truncations from one end of the polymer. Truncation from the opposite terminus of the median contact order structure and studies of the highest and lowest contact order structures produce effectively identical results (data not shown).

\section{Acknowledgements}

The authors thank Marek Cieplak for motivating this work with his careful studies of the determinants of Go polymer folding rates and for much stimulating correspondence. We gratefully acknowledge critical commentary from JoanEmma Shea, Eric Sorin and Laura Bartley. This work was supported by NIH grant R01GM6286801A2.

\section{References}

1. Bryngelson, J. D. \& Wolynes, P. G. (1989). Intermediates and barrier crossing in a random-energy model (with applications to protein folding). J. Phys. Chem. 93, 6902-6915.

2. Sali, A., Shacknovich, E. \& Karplus, M. (1994). How does a protein fold? Nature, 369, 248-251.

3. Klimov, D. K. \& Thirumalai, D. (1996). Criterion that determines the foldability of proteins. Phys. Rev. Lett. 76, 4070-4073.

4. Sali, A., Shakhnovich, E. \& Karplus, M. (1994). Kinetics of protein-folding: a lattice model study of the requirements for folding to the native state. J. Mol. Biol. 235, 1614-1636.

5. Abkevich, V. I., Gutin, A. M. \& Shakhnovich, E. I. (1995). Impact of local and non-local interactions on thermodynamics and kinetics of protein-folding. J. Mol. Biol. 252, 460-471.

6. Onuchic, J. N., LutheySchulten, Z. \& Wolynes, P. G. (1997). Theory of protein folding: the energy landscape perspective. Annu. Rev. Phys. Chem. 48, 545-600.
7. Gillespie, B. \& Plaxco, K. W. (2000). Non-glassy kinetics in the folding of a simple, single domain protein. Proc. Natl Acad. Sci. USA, 97, 12014-12019.

8. Millet, I. S., Townsley, L., Chiti, F., Doniach, S. \& Plaxco, K. W. (2002). Equilibrium collapse and the kinetic "foldability" of proteins. Biochemistry, 41, 321-325.

9. Plaxco, K. W., Simons, K. T. \& Baker, D. (1998). Contact order, transition state placement and the refolding rates of single domain proteins. J. Mol. Biol. 277, 985-994.

10. Plaxco, K. W., Simons, K. T., Ruczinski, I. \& Baker, D. (2000). Sequence, stability, topology and length; the determinants of two-state protein folding kinetics. Biochemistry, 39, 11177-11183.

11. Gromiha, M. M. \& Selvaraj, S. (2001). Comparison between long-range interactions and contact order in determining the folding rate of two-state proteins: application of long-range order to folding rate prediction. J. Mol. Biol. 310, 27-32.

12. Zhou, H. Y. \& Zhou, Y. Q. (2002). Folding rate prediction using total contact distance. Biophys. J. 82, 458-463.

13. Makarov, D. E. \& Plaxco, K. W. (2002). The topomer search model; a quantitative, first principles description of two-state protein folding kinetics. Protein Sci. In press.

14. Abe, H. \& Go, N. (1981). Noninteracting local-structure model of folding and unfolding transition in globular proteins. II. Application to two-dimensional lattice proteins. Biopolymers, 20, 10113-11031.

15. Koga, N. \& Takada, S. (2001). Roles of native topology and chain-length scaling in protein folding: a simulation study with a Gō-like model. J. Mol. Biol. 313, 171-180.

16. Cieplak, M. \& Hoang, T. X. (2002). Universality classes in folding times of proteins. Biophys. J. In press.

17. Eastwood, M. P. \& Wolynes, P. G. (2001). Role of explicitly cooperative interactions in protein folding funnels: a simulation study. J. Chem. Phys. 114, 4702-4716.

18. Flanagan, J. M., Kataoka, M., Shortle, D. \& Engelman, D. M. (1992). Truncated staphylococcal nuclease is compact but disordered. Proc. Natl Acad. Sci. USA, 89, 748-752.

19. Ladurner, A. G., Itzhaki, L. S., Gay, G. D. \& Fersht, A. R. (1997). Complementation of peptide fragments of the single domain protein chymotrypsin inhibitor 2. J. Mol. Biol. 273, 317-329.

20. Camarero, J. A., Fushman, D., Sato, S., Giriat, I., Cowburn, D., Raleigh, D. P. \& Muir, T. W. (2001). Rescuing a destabilized protein fold through backbone cyclization. J. Mol. Biol. 308, 1045-1062.

21. Shea, J. E., Onuchic, J. N. \& Brooks, C. L. (1999). Exploring the origins of topological frustration: design of a minimally frustrated model of fragment B of protein A. Proc. Natl Acad. Sci. USA, 96, 12512-12517.

22. Debe, D. A., Carlson, M. J. \& Goddard, W. A. (1999). The topomer-sampling model of protein folding. Proc. Natl Acad. Sci. USA, 96, 2596-2601.

23. Plotkin, S. S., Wang, J. \& Wolynes, P. G. (1997). Statistical mechanics of a correlated energy landscape model for protein folding funnels. J. Chem. Phys. 106, 2932-2948.

24. Chan, H. S., Bromberg, S. \& Dill, K. A. (1995). Models of cooperativity in protein-folding. Philos. Trans. R. Soc. B, 348, 61-70. 
25. Shimizu, S. \& Chan, H. S. (2002). Anti-cooperativity and cooperativity in hydrophobic interactions: three-body free energy landscapes and comparison with implicit-solvent potential functions for proteins. Proteins: Struct. Funct. Genet. 48, 15-30.

26. Kaya, H. \& Chan, H. S. (2002). Towards a consistent modeling of protein thermodynamic and kinetic cooperativity: how applicable is the transition state picture to folding and unfolding? J. Mol. Biol. 315, 899-909.

27. Fernandez, A., Colubri, A. \& Berry, R. S. (2002). Three-body correlations in protein folding: the origin of cooperativity. Physica A, 307, 235-259.

28. Fernandez, A. (2001). Protein folding cooperativity in the correlated lattice. Phys. Lett. A, 290, 101-105.
29. Chan, H. S. \& Dill, K. A. (1993). Energy landscapes and the collapse dynamics of homopolymers. J. Chem. Phys. 99, 2116-2127.

30. Verdier, P. H. \& Stockmayer, W. H. (1962). Monte Carlo calculations on dynamics of polymers in dilute solution. J. Chem. Phys. 36, 227-235.

31. Madras, N. \& Sokal, A. D. (1987). Nonergodicity of local, length-conserving Monte-Carlo algorithms for the self-avoiding walk. J. Stat. Phys. 47, 573-595.

32. Faisca, P. F. N. \& Ball, R. C. (2002). Topological complexity, contact order, and protein folding rates. J. Chem. Phys. 117, 8587-8591.

Edited by C. R. Matthews

(Received 24 September 2002; received in revised form 8 November 2002; accepted 18 November 2002)

Note added in proof: Faisca and Ball have very recently reported that Miyazawa-Jernigan type lattice polymers of length 54-80 monomers exhibit modestly topology-dependent folding rates, but that shorter polymers do not. ${ }^{32}$ We presume that the addition of cooperativity to these polymers would increase still further the topology dependence of their folding rates. 